"Autumn", by Dame Laura Knight, is the first of three valiant and not unsuccessful attempts at rendering atmospheric effects of great difficulty.

The same room contains several pictures by younger artists, two of whom appear for the first time : "A Yellow Dressing Table" is by Pat Newton, and "Still Life" by Henry Kilvert. There are also three flower pieces, "Autumn Flowers", by Elsie Atkins, who exhibited in 1944 and 1946 ; "Summer Flowers", by Vera Waddington, also an exhibitor in 1944 and 1946 ; and "Geraniums", by D. Travers-Smith, whose picture last year was, I think, her first. Both Miss Atkins and Miss Waddington paint flowers well, and each has shown in previous years that she is not afraid of other subjects. The arrangement of Miss Travers-Smith's geranium is rather obvious, and the design is over-emphasized by the background. Best of all is the yellow dressing-table in its garret window ; there is more originality in the subject, and more character in the painting.

One of Mr. Churchill's pictures hangs near the dressing-table. It is "Winter Sunshine", a not altogether successful sketch of a red-brick house standing in a snowy garden. The arrangement of the high lights, and the mass of shadow in the middle of the canvas, give the impression that the centre of the picture has dropped out. His other picture, said to be a later work, is in Gallery III. "The Loup River, Alpes Maritimes" is above the average in its rendering of flowing water. Taken together, they show Mr. Churchill to have considerable talent as a painter, but they display none of that robustiousness popularly associated with Mr. Churchill the politician.

If the Academy were restricted to oils, one might well stop there, for what has been said serves as a sample of the whole. As it is, there are other media to consider, and of them the water-colours in the South Rooms are easily the most important. Watercolour drawing is so essentially an English art that one is not surprised when its practitioners prove to be more consistently interesting than the painters in oils. R. Vivian Pitchforth, Barbara Jones, Hadley Rowe and Peter Lowry each has something worth saying, though Mr. Rowe should know better than to put tropical shells on what appears to be a typically English beach. Mr. Pitchforth is, of course, one of our leading water-colourists and an associate of the Academy, but Miss Jones and Mr. Rowe are, I believe, exhibiting for the first time. There is both character and individuality in their work. Mr. Lowry had pictures hung in 1945 and again last year; that he tends to dramatize his subject is not necessarily a fault. Two pictures of the shipyards at Appledore possess a maturity and sureness of touch which prove Michael Leszczyński to be much more than a beginner, even though this is the first time he has been hung in the Academy. Hal Yates" "Runswick" and Dennis James' "Snow Scene, Kingsley Way" each deserve more than a passing glance; the latter is not hung as well as it should be.

To return to the oil-paintings. Henry Hoyland has been represented by a picture or two since 1944, and, as his two studies of the circus show, he improves each year. Jean Primrose has two works of great promise in "Ballerina" and "Angela Reading", and that brings us to the Black and White Room (Gallery IX). This I found dull apart from Miss Winifred Austen's bird studies, which have a charm all their own, and Robert Austin's engravings. Sir Lionel Lindsay's dry-point is of particular interest: it throws a flood of light on his book "Addled Art".
There is little to be said about the architectural exhibits; present conditions are not kind, and architects are in partial eclipse. Neither is the sculpture very interesting. It is always difficult to make a gallery of small busts look anything but spotty, and this year the difficulties appear to have proved greater than usual.

Nevertheless, this is a 'good' Academy, and if the next can improve on it as much as this one has improved on last year's, then there will be firm reason for thinking that artists as a whole have recovered from their post-war malaise.

\section{THE ADRENAL CORTEX}

$T$ HE fourth meeting of the Society of Endocrinology, held on March 28 in the Department of Anatomy, University of Birmingham, was devoted to a discussion on the adrenal cortex. In the opening paper, entitled "Recent Advances in the Chemistry of the Adrenal Cortex", Dr. C. W. Shoppee mentioned briefly the six steroids newly isolated from adrenal tissue during the past seven years, which include œstrone, $\Delta^{4}$-androstene-3:17 dione, and 17-hydroxyprogesterone. The total number of adrenal steroids with known structures is now twenty-eight, of which six are active in preserving the life of the adrenalectomized animal. The structures of most of these compounds are completely determined. Where hydroxyl groups exist at positions 3 and 11 they are ( $\beta$ ) orientated, while hydroxyl groups at position 17 are almost certainly in the $(\alpha)$ position. During the past few years it has become clear that adrenal steroids, as well as deoxycholic acid, have the side-chain at position 17 in the $(\beta)$ position and not, as was previously believed, $(\alpha)$ orientated. Reichstein has now prepared all six physiologically active steroids artificially. The molecular features essential for high activity appear to be a 3-keto- $\Delta^{4}$-grouping, together with a $17(\beta)$ sidechain of the form $-\mathrm{CO} . \mathrm{CH}_{2} \mathrm{OH}$. For activity with respect to carbohydrate and protein metabolism, an oxygen atom at $\mathrm{C}_{11}$, either as a keto-group or a ( $\beta$ )-orientated hydroxyl group, appears to be essential.

The pure crystalline substances isolated from cortical extracts represent only a small fraction of the activity of the concentrates from which they are prepared: the greater part of the activity (about 90 per cent) remains in the so-called amorphous fraction. The latter shows somewhat anomalous physiological properties, and it is likely that there remain to be isolated one or more unknown labile compounds, probably containing five oxygen atoms, which are more active than any of the hormones yet obtained in a state of purity. Lowenstein and Zwemer (Endocrinology, 39, 63; 1946) have recently reported the isolation of 11-dehydrocorticosterone and two incompletely characterized substances, the combined activity of which equalled 80 per cent of the activity of the original concentrate. One of these compounds gave ascorbic acid by mild anaerobic hydrolysis; the other, presumably steroid, product of hydrolysis has not been isolated.

In his communication entitled "The PituitaryAdrenal Relationship", Dr. M. Reiss discussed first the problem of the isolation and biological assay of pituitary corticotrophic fractions. For assay purposes, Dr. Reiss and his colleagues have investigated the histological changes occurring in the adrenal glands of hypophysectomized rats. The sudanophilic 
substances between the zona glomerulosa and the zona fasciculata were found to disappear seven to ten days after hypophysectomy, the wide so-called sudanophobic zone thus formed being an enlargement of a very thin zone normally present in young rats. This zone is abolished by treatment of the hypophysectomized rat with corticotrophic extract, and a standardization of the extract is based on these histological changes. Workers in two different laboratories (those of H. M. Evans and of C. N. H. Long) have isolated, by different procedures, the same pure substance which they claim to be the corticotrophic hormone. This does not exclude the possible existence of more than one corticotrophic substance; and indeed when one considers the multitude of active substances produced by the adrenal cortex, the existence of different corticotrophic substances responsible for the mobilization of these adrenal steroids is by no means impossible. The influence of the known corticotrophic substance on the adrenal cortex is complex. The cholesterol content falls in the first three hours (rat) and later rises. A fall of ascorbic acid content is detectable an hour after corticotrophic treatment, and the fall is proportional to the dose given. By direct estimation the hormone content of the adrenal gland was found to be increased $24 \mathrm{hr}$. after treatment, but substantially reduced $48 \mathrm{hr}$. after (Hempill and Reiss, unpublished). The changes in the hormone activity of the blood were opposite to those of the adrenal tissue.

Although adrenal function is considerably diminished after hypophysectomy, there is no reason to believe that adrenal-cortical activity ceases in the absence of pituitary corticotrophin; the hypophysectomized rat can survive the avulsion a year or more, but it would die in the course of a few days if all the cortical tissue had been removed. Little is known about the nervous control of the pituitary anterior lobe production of corticotrophin, though it appears probable that the secretion of corticotrophin is influenced by a psychic factor. Although the physiological effects of corticotrophin are mainly to be ascribed to stimulation of the secretory activity of the adrenal cortex, it would not be surprising if some actions of corticotrophic extracts failed to agree with those of known adrenal steroids. It is probable that adrenal steroids remain to be isolated, while it is yet uncertain if all those isolated are of physiological significance.

In his survey of the "Adrenal Cortex and Metabolism (including Electrolyte Metabolism)", Prof. F. G. Young pointed out that at present no simple comprehensive theory seems able to explain the diverse actions of adrenal steroids, and as more experimental evidence becomes available the earlier generalizations seem less and less certain. It is important not to neglect the fact that when one injected a hormone one was studying not merely the effect of the hormone on the animal but also the interaction of the animal and the hormone. Changes in food intake, adaptation to newly imposed experimental procedures particularly in long-term procedures, metabolic interconversions of administered steroid substances, all could and probably do complicate the interpretation of apparently simple experiments. In particular, the control of food intake by paired-feeding control experiments is of the greatest importance. Conway and Hingerty (Biochem. $J ., 40,561 ; 1946)$ have shown that the potassium ion content of the muscles of the adrenalectomized rat is raised. This controverts the idea that the increased blood potassium is to be ascribed to leakage of potassium ions from the tissue cells. These authors have also shown a striking increase in plasma and muscle magnesium ion content, possibly significant to the muscular asthenia of adrenal insufficiency. The influences of adrenalectomy on carbohydrate and protein metabolism can be largely prevented by adequate therapy with sodium chloride, although such treatment does not prevent the catastrophic fall in carbohydrate storage which results from a short period of starvation in the adrenalectomized rat. Since deoxycorticosterone and the amorphous fraction of adrenal extracts both possess striking activity in inducing retention of sodium chloride in the normal and adrenalectomized animal, it is perhaps surprising that these adrenal fractions have so little action on carbohydrate and protein metabolism. Moreover, an important in vitro action of adrenal extract on hexokinase activity has been traced to the amorphous fraction.

Cori et al. (J. Biol. Chem., 160, 633; 1945 : Fed. Proc., 5, 150; 1946) have shown that the activity of hexokinase in catalysing the transfer of a phosphate group from adenosinetriphosphate to glucose, forming glucose-6-phosphate, is diminished in vitro by the addition of anterior pituitary extract, and that this pituitary inhibition can be emphasized and extended by the addition of adrenal extract, though the latter alone has no obvious action in this respect. The influence in vivo of adrenal extract on other enzyme systems (for example, arginase, phosphatase, cytochrome oxidase) has opened up new lines of investigation of the greatest significance. It seems possible that the adrenal hormones, like enzymes, are not essential for the mechanisms of the body, but hasten or retard reactions which will proceed in the absence of the hormones.

In discussing "The Adrenal Cortex in Shock and Stress", Dr. G. Ungar pointed out that there is good experimental evidence for three main conclusions : (1) adrenalectomized animals exhibit a lowered resistance to many different types of noxious stimuli ; (2) the adrenal glands show- morphological changes after exposure to these stimuli; (3) the administration of adrenal steroids can, in some instances, enhance the resistance of adrenalectomized animals to these stimuli. It has long been known that stimuli can sometimes induce in animals a temporary increase in resistance to the same and non-related stimuli. Selye has proposed the term 'adaptation syndrome' in this connexion, and divides this syndrome into three stages: the alarm reaction, the adaptation phase, and the exhaustion phase. Most of the symptoms of the adaptation phase cannot be induced in adrenalectomized animals, though the secretory activity of the adrenal cortex is not the only factor concerned. The influence of adrenal steroids on carbohydrate and protein metabolism, and on the involution of the thymus and lymphatic tissue generally, are of importance, but, as Dr. Ungar himself has shown, the spleen is also concerned. Noxious stimuli cause shortening of bleeding time, increase in capillary resistance, and reduction of capillary permeability. All these effects are prevented by hypophysectomy, or adrenalectomy, or splenectomy, and can be reproduced in appropriate circumstances by corticotrophin, whole adrenal extract and suitable spleen extract.

A substance which can account quantitatively for the action of cortical extracts is a recently isolated 
adrenal steroid, $\Delta^{4}$-pregnene-3-one-17( $\left.\beta\right): 20 \beta: 21$ triol. The active splenic substance has been obtained in very pure form, and its chemical identification is being carried out by Prof. E. L. Hirst in Manchester. During adaptation there is a reduction in the amount of histamine that can be liberated from blood cells. Histamine is liberated through the action of a proteolytic enzyme normally held in check by an inhibitor; in certain pathological conditions the enzyme is set free and is then able to split off histamine from some protein substrate. It is possible that the adrenal cortex can inhibit the liberation of the proteolytic enzyme.

In her consideration of the rate of secretion of the mammalian suprarenal cortex, Dr. Marthe Vogt first discussed the different methods available for the biological assay of adrenal cortical hormones. Most of the information on the amounts of cortical hormones present in body fluids has been obtained with methods involving the deposition of glycogen in the livers of fasting normal rats, or involving the protection of adrenalectomized rats against the effects of a low environmental temperature (Selye and Schenker assay). Dr. Vogt has successfully applied the second of these methods to the estimation of hormone activity in the suprarenal blood of experimental animals. The results were expressed, by comparison with commercial extracts prepared from slaughter-house adrenal glands, in terms of the amounts of slaughter-house material required for the preparation of crude extract sufficient to give the observed physiological activity. On this basis the normal output of adrenal hormones in a $10-\mathrm{kgm}$. dog is $720 \mathrm{gm}$. $/ \mathrm{hr}$., which is approximately equivalent to $54 \mathrm{mgm}$./day of 17-hydroxy-11-dehydrocorticosterone.

These results confirm the idea that the hormones are not stored in the gland in the active form to any appreciable extent, but are produced from an inactive precursor and immediately released into the circulation. Furthermore, since arterial or heart blood contains no significant activity, it appears that the secreted steroids are rapidly inactivated in the tissues. The rate of hormone secretion is greatly increased by stimulation of the splanchnic nerves, and since this effect can be duplicated by the intravenous infusion of adrenaline, it appears to be an indirect one via the adrenal medulla. It has been found possible to assay the adrenal steroids liberated in the isolated perfused adrenal gland. In such a perfused preparation a prolonged increase in the rate of secretion can be induced by the addition of corticotrophin to the circulating blood. In the absence of any such added stimuli, the isolated glands have been found to secrete continuously at a steady rate which lies within the limits observed in the experiments on the whole animal. Experiments of this type are obviously of great value, since the conditions are much more experimentally controllable than those in experiments on the intact animal.

In the course of his summing-up of the discussion, the chairman (Prof. S. Zuckerman) pointed out the paradox that although extra dietary sodium chloride will maintain an adrenalectomized animal in good health under controlled environmental conditions, the secretory activity of the adrenal glands is normally surprisingly high and can rise to very high levels under the influence of environmental stimuli. What is the importance to the body of this normal secretory activity of the adrenal glands, and what is the fate of the secreted steroids? Although the adrenal secretion may not be essential for survival under some conditions, and does not necessarily initiate reactions in the body but hastens or retards enzymically controlled processes, it is important not to under-estimate the importance of adrenal hormones, particularly under conditions of stress. The present multiplicity of apparently unrelated experimental data does not yet allow any simple generalization about the function of adrenal hormones in general ; but the importance of correlating observa. tions which can accurately be correlated in a limited way should not be neglected.

\section{OBITUARIES}

\section{Dr. S. Monckton Copeman, F.R.S.}

Sydney Arthur Monckton Copeman, the eldest son of the Rev. Canon A. C. Copeman, was born at Norwich on February 21, 1862, and was educated at King Edward VI School, Norwich, and at Corpus Christi College, Cambridge. After obtaining a second class in the Natural Sciences Tripos in 1882, he went to St. Thomas's Hospital and took the M.B. Cambridge in 1886. He proceeded to the M.D. in 1890 and was elected F.R.C.P. in 1899. For a short time he was assistant lecturer on physiology and morbid histology at St. Thomas's. In 1891 he became a medical inspector of the Local Government Board, and all his active medical life was spent in the service of that Department and, afterwards, as a medical officer of the Ministry of Health. In the same year he was one of the founders of the Medical Research Club.

Medical research early engaged Copeman's attention, and among his early papers was one on the specific gravity of the blood in disease and another on hæmatoporphyrin in urine. It is, however, by his work on variola and vaccinia that he established his scientific reputation.

The early experiments of Ceely (1839-40), of Badcock (1840) and of Klein (1892) had demonstrated that typical vaccinia may be produced in the calf by the inoculation of variolous matter. In 1892 Copeman showed that it was possible to inoculate calves with variolous lymph taken from a girl suffering from smallpox, that the inoculated calves showed vesiculation, that the results obtained became greatly modified in the course of successive removes, and further, that animals which had been thus treated were no longer susceptible to vaccination. In the same year he proved that rhesus monkeys are susceptible not only to vaccination but also to smallpox. He next investigated the degree of protection against smallpox afforded in the monkey by previous vaccination and the protection against vaccina. tion afforded by variolation; and went on to com. pare the effect produced by the use of human and of calf vaccine respectively. By passing smallpox virus through monkeys and then vaccinating calves from the monkeys, he produced typical vaccinia, which gave excellent strains of vaccine lymph.

In 1865 a Commission, appointed by the Society of Medical Sciences at Lyons, having Chauveau at its head, had reported to the effect that cowpox and smallpox were dual diseases. Copeman's work supported the view that cowpox and horsepox are variola modified by transmission, or, as he phrased it: "Smallpox and vaccinia are both of them descended from a common stock-from an ancestor, for instance - which resembled vaccinia far more than it resembled smallpox". 